\title{
determinación de sillice libre en materias primas para la industria del cemento
}

\author{
J. CALLeJA* y B. BACLE**
}

\section{resumen}

La existencia de sílice libre en las materias primas y, por lo tanto, en los crudos empleados en la fabricación de cemento Portland es desventajosa por la escasa reactividad de esta sílice, sobre todo si no posee una finura suficiente.

La determinación de sílice cuarzosa en tales materiales es decisiva para la buena marcha de la clinkerización en fábricas cuyas materias primas adolecen de la desventaja de contenerla.

Los métodos propugnados suelen ser poco reproducibles y precisos, y dan resultados bastante erráticos.

En el presente trabajo, y después de haber ensayado diversos métodos citados en la bibliografía, se describe y recomienda un procedimiento, basado en el ataque del material por ácido pirofosfórico.

Las variables que juegan en el proceso descrito son el tamaño de grano de la muestra, en particular el de la sílice libre, la temperatura y el tiempo de ataque. En las condiciones que se especifican se consigue una disolución prácticamente total de los silicatos, sin que apenas se disuelvan las partículas más finas de cuarzo.

Con ello se consigue una exactitud y una precisión tal como pone de manifiesto el análisis estadístico de los resultados obtenidos al determinar sílice libre en diversas materias primas y crudos para cemento.

\section{- importancia técnica del problema}

Cuando se estudia la dosificación de un crudo en una fábrica de cemento para obtener un clínker, los tantos por ciento de los constituyentes de éste se calculan a base del análisis quimico de aquél. La sílice total de un crudo puede ser de dos clases muy diferentes desde el punto de vista de la reactividad frente a la cal en el proceso de clinkerización: la sílice que

\footnotetext{
* Doctor en Ciencias Químicas. Jefe del Departamento de Química del Instituto "Eduardo Torroja" de la Construcción y del Cemento del Patronato "Juan de la Cierva" de Investigación Técnica del Consejo Superior de Investigaciones Científicas,

* Perito Industrial Químico, de la sección de Análisis de dicho Departamento.
} 
se encuentra formando parte de los silicatos de la arcilla, fácilmente reactiva, y la sílice en forma de cuarzo, mucho menos reactiva, que puede acompañar tanto al componente calizo como al componente arcilloso.

Con los métodos analíticos usualmente aplicados a las materias primas para cemento (en los cuales se procede por fusión alcalina) se determina el contenido de sílice total, suma de las dos consideradas, sin hacer distinción entre ellos. Sin embargo, dada su distinta reactividad, a la hora de combinarse con la cal para formar silicatos, la sílice cuarzosa puede hacerlo parcialmente, pero en cualquier caso es difícil prever la medida en que lo hace.

Al depender de múltiples variables la reactividad de la sílice cuarzosa —grado de finura, temperatura y tiempo de reacción, fundamentalmente - no es fácil que los módulos - hidráulico y silícico- y el grado de saturación reales de un clínker coincidan con los previsibles según el crudo de partida, como tampoco los contenidos de cal libre y de residuo insoluble de dicho clínker serán, en general, los esperados.

Una forma de aproximar los resultados reales a los previstos consiste en hacer que prácticamente toda la sílice reaccione, y para ello se debe aumentar el grado de división de las partículas de cuarzo y con él su reactividad, así como clinkerizar a temperatura suficientemente alta, y prolongar cuanto sea necesario el tiempo de reacción. De estas tres medidas, la primera es la más practicada, tanto por razones técnicas como económicas.

Una mezcla de cuarzo y cal, calentada en autoclave, reacciona - fundamento de la fabricación de ladrillos de cal y arena - y con tanta mayor rapidez y facilidad transcurre la reacción, cuanto mayor es la superficie presentada por el cuarzo.

En las citadas condiciones un cristal de cuarzo de $12,8 \mathrm{~g}$ pierde durante 48 horas a $180^{\circ} \mathrm{C}$ el $0,25 \%$ de su peso, mientras que con un tamaño de grano de $0,15 \mathrm{~mm}$ la pérdida de peso es del $12,4 \%$. Si el cuarzo se reduce a polvo impalpable puede convertirse en ácido silícico hidratado coloidal mediante una ebullición prolongada en agua (1).

La gran influencia que en general tiene la finura del crudo en la perfecta cocción del clínker es de sobra conocida: cuanto mayor sea dicha finura, más fácil será la cocción y más baja la temperatura necesaria para lograr un clínker de buena calidad, siendo económicamente preferible en muchos casos el aumento del grado de finura al aumento de la temperatura de cocción. Un aumento de $100^{\circ} \mathrm{C}$ en la temperatura de cocción, de 1.400 a $1.500^{\circ} \mathrm{C}$, produce un incremento en el consumo de calor de unas 60 calorías por kilogramo de clínker, lo cual corresponde por término medio a una elevación del 4 al $5 \%$ en el gasto de combustible (2).

El contenido de cal libre de un clínker obtenido a partir de un crudo con sílice cuarzosa se reduce considerablemente, para una temperatura y una dosificación del crudo dadas, aumentando la superficie específica del cuarzo. Se logra pasar del 6 al 0,5 \% de cal libre, aproximadamente, mediante una disminución del tamaño de las partículas de cuarzo de 0,14 a $0,04 \mathrm{~mm}$ (3). Por lo tanto, para finuras elevadas, el cuarzo existente en un crudo puede considerarse como sílice fácilmente reactiva con los componentes básicos de la mezcla sin que impida obtener un clínker de buena calidad, con un mínimo contenido de cal libre y de residuo insoluble.

Las partículas gruesas de sílice no reaccionan en las condiciones en que se efectúa la cocción en los hornos de las fábricas de cemento, ya que la cal no tiene tiempo de penetrar por difusión hacia el interior del grano de cuarzo. Juega un papel de primer orden la mezcla in- 
tima de los componentes del crudo (4), puesto que la constilución y calidad de un clínker está regida por la composición de la masa "homogénea" que lo engendra, y no por la composición "media" de esta masa, cuando no es homogénea. Cuanto más se aproxime el crudo a una mezcla molecular por aumento del grado de división de los constituyentes, tanto mejor se conseguirá su combinación por contacto, sin intervención de la difusión, siendo también menor la necesidad de fundentes (כ).

Los ensayos realizados muestran que en un crudo no debe existir más del $0,5 \%$ de partículas de cuarzo de tamaño superior a $0,2 \mathrm{~mm}$, ni más del $1 \%$ de tamaño comprendido entre $0,09 \mathrm{~mm}$ y $0,2 \mathrm{~mm}$, para lograr una buena formación de los constituyentes del clínker. Esstudios más recientes indican que los crudos con un grado de saturación de cal de $90 \%$ soportan el $1 \%$ de partículas de cuarzo de $0,2 \mathrm{~mm}$ sin gran aumento en el contenido de cal libre (2).

De las consideraciones expuestas se desprende la importancia que tiene el conocimiento de la cantidad de sílice cuarzosa presente en las materias primas para la fabricación del clínker.

\section{-método analítico adoptado para la determina- cioun de la sílice cuarzosa}

Después de un estudio minucioso de los métodos existentes para la determinación de la sílice cuarzosa, (6), (7), (8), (9), (10) y (11), y de la puesta en práctica de buena parte de ellos, se llegó a la forma operatoria que a continuación se describe.

Se realiza la determinación en una muestra con un tamaño de grano comprendido entre los tamices de 43 y 88 micras de luz de malla. Esto se justifica por el hecho de que los crudos en la industria del cemento se muelen a un grado de finura correspondiente a un residuo de alrededor del $14 \%$ sobre el tamiz de 88 micras $\left(4.900\right.$ mallas $\left./ \mathrm{cm}^{2}\right)$, y por juzgar como fácilmente reactivas con el componente calizo, en las condiciones normales de un horno, las partículas de cuarzo de tamaño inferior a 43 micras (3), pudiendo por ello considerarse este cuarzo como sílice semejante en su comportamiento a la de los silicatos de la arcilla, por las razones expuestas anteriormente.

\section{1. fundamento del método}

El método es gravimétrico y se basa en la disolución, por la acción del ácido pirofosfórico, de los silicatos presentes en la muestra, y en la determinación, por pesada, del residuo restante constituido por el cuarzo. En detalle consiste en lo siguiente:

Se pesan en un crisol de porcelana 0,5 gramos de muestra representativa, con un tamaño de grano comprendido entre 0,088 y $0,043 \mathrm{~mm}$, previamente desecada a $105-110^{\circ} \mathrm{C}$. Se calcina a $850-900^{\circ} \mathrm{C}$ durante 15 minutos. Este tratamiento es conveniente para conseguir un ataque posterior más rápido de los silicatos.

En un vaso de cuarzo, de 100 a $150 \mathrm{ml}$ de capacidad, se ponen $20 \mathrm{ml}$ de ácido ortofosfórico de $85 \% \mathrm{~d}=1,710$, y se calienta rápidamente para convertirlo en pirofosfórico. Esto se consigue al alcanzar una temperatura comprendida entre 220 y $250^{\circ} \mathrm{C}$, lo que se $\operatorname{logra}$, 
aproximadamente, al cabo de 10 minutos, durante los cuales debe tenerse en cuenta que se producen proyecciones de ácido íuera del vaso, al desprenderse el agua que se libera en el paso del ácido ortofosfórico a pirofosfórico.

Cuando la ebullición se atenúa y sólo se desprende alguna burbuja del fondo del vaso, se separa éste del foco de calefacción para que se enfrie. Una vez frío, aproximadamente a $70^{\circ} \mathrm{C}$, se añade la muestra directamente del crisol, donde ha sido tratada de la forma antes expuesta, procurando que se reparta por la superficie del ácido, y arrastrando todas las partículas que pueden quedar adheridas al crisol con ayuda de un pequeño pincel. A continuación se calienta de nuevo rápidamente, atacándose la muestra durante un total de 16 minutos, contados a partir del momento en que se sitúa el vaso sobre la superficie de calefacción (en las determinaciones realizadas esta superficie de calefacción era capaz de elevar, desde la temperatura ambiente hasta la temperatura de ebullición, $200 \mathrm{ml}$ de agua contenida en un vaso de $250 \mathrm{ml}$, de forma alta, en un tiempo de 15 minutos). Durante el ataque de la muestra conviene agitar el vaso de vez en cuando, sujetándolo con los dedos protegidos por dos trozos de tubo de goma e imprimiendo al líquido un movimiento de rotación. Hay que procurar qque no quede por las paredes muestra sin atacar, pues generalmente la muestra flota en el ácido y, a medida que va siendo atacada, el cuarzo se deposita en el fondo del vaso. se debe cuidar de que la temperatura no sea excesiva, lo que se pone de manifiesto por el desprendimiento de humos blancos. Tampoco se debe prolongar excesivamente el ataque, porque ello originaría, además de la disolución de una parte de cuarzo, la formación de sílice gelatinosa que impediría la fácil filtración de los silicatos disueltos.

Una vez transcurridos los 16 minutos, se aparta el vaso del foco de calefacción y se deja enfriar a unos 50-70 $0^{\circ} \mathrm{C}$. Cuando está lo suficientemente frío --puede ayudarse al enfriamiento sumergiendo el fondo del vaso en agua fría - se vierte cuidadosamente su contenido, agitando al mismo tiempo con una varilla - la reacción de dilución es exotérmica y existe el peligro de proyecciones- en un vaso de $150 \mathrm{ml}$ de capacidad, de forma alta, que contiene $70-80 \mathrm{ml}$ de agua destilada a punto de ebullición. En el agua se habrá echado, en pequeños trozos, la mitad de un disco de papel filtro exento de cenizas, de $11 \mathrm{~cm}$ de diámetro, cuyos trozos tienen la misión de retener las partículas coloidales que puedan existir en el producto del ataque

Las últimas porciones de líquido y de cuarzo adheridas al vaso se arrastran con ayuda del chorro de un frasco lavador que contiene agua destilada a punto de ebullición. Una vez que se ha pasado todo al vaso con agua destilada, se calienta el conjunto a ebullición, agitando con la varilla durante 2 ó 3 minutos.

A continuación se filtra, lo más caliente posible y de forma continua, por papel de filtro sin cenizas, de porosidad media - Schleicher y Schüll, banda blanca, número $589^{2}$ o equivalente-, el cual se ha calentado previamente en su embudo con agua a punto de ebullición inmediatamente antes de comenzar la filtración. Es muy importante que todas estas operaciones se realicen a la temperatura más elevada posible, para que la filtración tenga lugar de forma satisfactoria. Filtrado el líquido y arrastrado el residuo cuarzoso al embudo, se lava éste con agua caliente hasta la desaparición de la acidez en las aguas de lavado, lo que debe comprobarse mediante papel indicador de $\mathrm{pH}$. El lavado debe efectuarse desde el borde de papel de filtro colocado en el embudo.

Después se deseca el papel de filtro con el residuo, se calcina y se pesa. La calcinación puede realizarse sobre la llama de un quemador que alcance los $900^{\circ} \mathrm{C}$, teniendo la precaución de que al principio no sea demasiado rápida la combustión del papel. 
El tanto por ciento de cuarzo en la muestra vendrá dado por la expresión:

$$
\text { cuarzo } \%=100 \cdot \frac{p_{2}-p_{1}}{p_{m}}
$$

en la que:

$$
\begin{aligned}
& p_{1}=\text { tara del crisol, previamente calcinado; } \\
& p_{2}=\text { peso del crisol, con el residuo calcinado: } \\
& p_{m}=\text { peso de la muestra empleada. }
\end{aligned}
$$

\section{2. causas de error}

El inconveniente principal del método propuesto, y que puede ser fuente de error en determinados casos particulares, estriba en la diferente solubilidad de los diversos silicatos que pueden entrar a formar parte de la muestra que se analiza. Como resultado de un estudio de esta diferente capacidad de los diversos silicatos para disolverse en ácido pirofosfórico, se aconseja hacer el ataque de la muestra durante un tiempo de 16 minutos, aunque los materiales arcillosos corrientemente empleados en la fabricación de cemento puedan ser disueltos en cinco minutos. Este aumento del tiempo de ataque es conveniente, no obstante, para que entren en disolución los feldespatos, que corrientemente son minerales que pueden acompañar a las arcillas o margas. Los feldespatos del tipo de la ortoclasa, albita y anortita, se disuelven prácticamente en un $99 \%$ de su totalidad, en 16 minutos de tratamiento según el método descrito. Este tratamiento de $\mathbf{1 6}$ minutos podrá ocasionar, aproximadamente, según demuestran los ensayos realizados, una disolución del 1,8\% del cuarzo presente - al estado de división de la muestra alcanzado según el método, esto es, para partículas comprendidas entre 43 y 88 micras - lo cual significa que si un crudo de cemento tiene un $10 \%$ de cuarzo, al hacer su determinación por el método descrito se podrá cometer un error probable, por defecto, de $10 \times 0,018=0,18 \%$, que puede considerarse despreciable.

\section{- resulfados}

Con el fin de determinar la bondad del método se prepararon muestras artificiales, añadiendo cantidades conocidas de cuarzo a muestras de arcillas, calizas y crudos de cemento.

\begin{tabular}{|c|c|c|c|c|c|c|c|c|c|}
\hline \multirow{2}{*}{$\begin{array}{c}\text { Muestra } \\
\text { núm. }\end{array}$} & \multicolumn{3}{|c|}{ ARCILLA } & \multicolumn{3}{|c|}{ CALIZA } & \multicolumn{3}{|c|}{ CIRUDO } \\
\hline & $\begin{array}{l}\text { Real } \\
(\%)\end{array}$ & $\begin{array}{l}\text { Hallado } \\
(\%)\end{array}$ & Dil. & $\begin{array}{l}\text { Real } \\
(\%)\end{array}$ & $\begin{array}{c}\text { Hallado } \\
(\%)\end{array}$ & Dif. & $\begin{array}{c}\text { Real } \\
(\%)\end{array}$ & $\underset{(\%)}{\text { Hallado }}$ & Dif. \\
\hline $\begin{array}{l}1 \\
2 \\
3 \\
4 \\
5 \\
6 \\
7 \\
8\end{array}$ & $\begin{array}{l}45,7 \\
47,1 \\
46,5 \\
45,9 \\
45,6 \\
46,0 \\
46,2 \\
46,0\end{array}$ & $\begin{array}{l}45,7 \\
47,1 \\
46,3 \\
45,7 \\
45,4 \\
45,7 \\
46,5 \\
45,4\end{array}$ & $\begin{array}{r}-0,0 \\
-0,0 \\
-0,2 \\
-0,2 \\
-0,2 \\
-0,3 \\
+0,3 \\
-0,6\end{array}$ & $\begin{array}{l}40,7 \\
41,2 \\
42,2 \\
41,8 \\
40,2 \\
43,1 \\
42,8 \\
42,5\end{array}$ & $\begin{array}{l}40,3 \\
40,7 \\
42,4 \\
41,4 \\
39,8 \\
43,0 \\
42,9 \\
42,3\end{array}$ & $\begin{array}{r}0,4 \\
-0,5 \\
+\quad 0,2 \\
-0,4 \\
-0,4 \\
-0,1 \\
+\quad 0,1 \\
0,2\end{array}$ & $\begin{array}{l}43,0 \\
45,7 \\
44,3 \\
44,7 \\
45,2 \\
44,5 \\
43,9 \\
44,7\end{array}$ & $\begin{array}{l}43,2 \\
46,1 \\
44,2 \\
44,8 \\
45,4 \\
44,6 \\
43,9 \\
44,5\end{array}$ & $\begin{array}{l}+0,2 \\
+0,4 \\
+0,1 \\
+0,1 \\
+0,2 \\
+0,1 \\
+0,0 \\
+0,2\end{array}$ \\
\hline
\end{tabular}
Las cantidades de cuarzo añadidas fueron considerablemente altas. Los resultados se dan en el cuadro 1 .

C U A D R O 1 
En el cuadro 2 se dan los resultados de las determinaciones del contenido de silice cuarzosa en materias primas empleadas en la industria del cemento, y se ponen de relieve las diferencias entre los valores absolutos máximo y mínimo hallados en 10 determinaciones para cada muestra.

C U A D R O 2

\begin{tabular}{|c|c|c|c|c|c|c|}
\hline \multirow{2}{*}{$\begin{array}{l}\text { Determinación } \\
\text { número }\end{array}$} & \multicolumn{6}{|c|}{$M U E S T R \quad A$} \\
\hline & $\underset{x}{\operatorname{Caliza}} 5$ & $\underset{x}{\text { Crudo } 4}$ & $\underset{x}{\text { Crudo } 3}$ & $\underset{x}{\operatorname{Arcilla}} 1$ & $\underset{x}{\underset{x}{\text { Crudo } \mathrm{H}}}$ & $\underset{x}{\text { Crudo } 2}$ \\
\hline $\begin{array}{r}1 \\
2 \\
3 \\
4 \\
5 \\
6 \\
7 \\
8 \\
9 \\
10\end{array}$ & $\begin{array}{l}1,69 \\
1,70 \\
1,70 \\
1,74 \\
1,78 \\
1,78 \\
1,79 \\
1,80 \\
1,86 \\
1,87\end{array}$ & $\begin{array}{l}5,05 \\
5,05 \\
5,08 \\
5,08 \\
5,12 \\
5,16 \\
5,17 \\
5,20 \\
5,23 \\
5,36\end{array}$ & $\begin{array}{l}5,02 \\
5,12 \\
5,14 \\
5,25 \\
5,27 \\
5,27 \\
5,30 \\
5,39 \\
5,39 \\
5,43\end{array}$ & $\begin{array}{l}6,82 \\
6,84 \\
6,90 \\
6,90 \\
6,92 \\
6,95 \\
7,10 \\
7,10 \\
7,20 \\
7,30\end{array}$ & $\begin{array}{l}7,65 \\
7,68 \\
7,674 \\
7,85 \\
7,85 \\
7,87 \\
7,87 \\
7,94 \\
7,95 \\
7,99\end{array}$ & $\begin{array}{l}8,92 \\
8,98 \\
9,00 \\
9,00 \\
9,10 \\
9,15 \\
9,15 \\
9,21 \\
9,25 \\
9,31\end{array}$ \\
\hline Medias $\bar{x}$ & 1,771 & 5,150 & 5,258 & 7,003 & 7,839 & 9,108 \\
\hline $\begin{array}{l}\text { Valores abs. máx. } \\
\text { Valores abs. mín. }\end{array}$ & $\begin{array}{l}1,87 \\
1,69\end{array}$ & $\begin{array}{l}5,36 \\
5,05\end{array}$ & $\begin{array}{l}5,43 \\
5,02\end{array}$ & $\begin{array}{l}7,30 \\
6,82\end{array}$ & $\begin{array}{l}7,99 \\
7,65\end{array}$ & $\begin{array}{l}9,31 \\
8,93\end{array}$ \\
\hline Diferencias & 0,18 & 0,31 & 0,41 & 0,48 & 0,34 & 0,38 \\
\hline
\end{tabular}

\section{- discusión de los resultados}

Con los resultados del cuadro 2 que, como puede apreciarse, se hallan ordenados en sentido creciente de izquierda a derecha y de arriba a abajo, se ha llevado a cabo un estudio estadístico que permite evaluar la calidad y características del método.

Con los $\mathrm{n}(=10)$ valores individuales $x$ hallados para cada uno de los seis materiales estudiados, $\mathrm{y}$ con sus medias aritméticas $\ddot{x}$, se han calculado en cada caso los siguientes parámetros estadísticos:

$$
\begin{aligned}
& \text { - las varianzas } v=\frac{\Sigma(x-\bar{x})^{2}}{n-1}, \\
& \text { - las desviaciones típicas } \sigma=\sqrt{v}, \\
& \text { - los coeficientes de la variación } \sigma \%=100 \frac{\sigma}{\bar{a}}, \\
& \text { - las desviaciones medias o errores típicos } \sigma_{m}=\frac{\sigma}{\sqrt{n}} .
\end{aligned}
$$

Los valores calculados se dan en el cuadro 3 : 
C U A D R O 3

\begin{tabular}{|c|c|c|c|c|c|c|}
\hline $\begin{array}{l}\text { Parámetros } \\
\text { estadísticos }\end{array}$ & Caliza 5 & Crudo 4 & Crudo 3 & Arcilla 1 & Crudo $\mathrm{H}$ & Crudo 2 \\
\hline $\begin{array}{c}v \\
\sigma \\
\sigma \% \\
\sigma_{m}\end{array}$ & $\begin{array}{l}0,004 \\
0,063 \\
3,56 \\
0,02\end{array}$ & $\begin{array}{l}0,009 \\
0,095 \\
1,84 \\
0,03\end{array}$ & $\begin{array}{l}0,017 \\
0,130 \\
2,47 \\
0,04\end{array}$ & $\begin{array}{l}0,026 \\
0,162 \\
2,31 \\
0,05\end{array}$ & $\begin{array}{l}0,013 \\
0,114 \\
1,46 \\
0,04\end{array}$ & $\begin{array}{l}0,016 \\
0,126 \\
1,39 \\
0,04\end{array}$ \\
\hline
\end{tabular}

Los valores de $\sigma$ permiten, a su vez, determinar si entre los resultados experimentales del cuadro 2 hay alguno estadísticamente aberrante y, por lo tanto, estadísticamente excluible del cálculo de las medias aritméticas.

Para ello se calcula el limite de confianza $\bar{x} \pm \sigma t$, utilizando el coeficiente $t$ de Fischer (12) para una probabilidad de $95 \%$, y para un valor $N=n-1=9$, correspondiente al número de determinaciones $n$, que en el caso presente es 10 . El valor de $l$ es 2,262.

Cualquier valor experimental del cuadro 2 tiene, en cada caso, el $95 \%$ de probabilidades de estar comprendido entre los límites:

$$
\bar{x} \pm \sigma t \text {. }
$$

es decir, entre los límites indicados en el cuadro 4.

C U A D R O 4

\begin{tabular}{|c|c|c|c|c|c|c|}
\hline 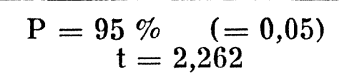 & Caliza 5 & Crudo 4 & Crudo 3 & Arcilla 1 & Crudo $\mathrm{H}$ & Crudo 2 \\
\hline Limites: & & & & & & \\
\hline Superior & 1,91 & 5,36 & 5,55 & 7,37 & 8,10 & 9,39 \\
\hline Inferior & 1,63 & 4,94 & 4,97 & 6,63 & 7,58 & 8,83 \\
\hline
\end{tabular}

La comparación de estos límites con los valores máximos y mínimos del cuadro 2 permite afirmar que en ningún caso se dan resultados aberrantes, y que todos los resultados pueden y deben entrar en el cálculo de las respectivas medias aritméticas.

Del mismo modo, el límite de confianza de la media verdadera es $\bar{x} \pm \sigma_{m} t$, es decir, ésta tiene el $95 \%$ de probabilidades de estar comprendida entre los límites:

$$
\bar{x} \pm \sigma_{m} t
$$

lo que supone un error relativo en la media aritmética con respecto a la media verdadera de:

$$
\varepsilon \%=100 \frac{\sigma_{m} t}{\bar{x}}
$$

Los valores correspondientes se dan en el cuadro 5. 
C U A D R O 5

\begin{tabular}{|c|c|c|c|c|c|c|}
\hline $\begin{array}{c}\mathrm{P}=95 \%,(=0,05) \\
\mathrm{t}=2,262\end{array}$ & Caliza 5 & Crudo 4 & Crudo 3 & Arcilla 1 & Crudo $\mathrm{H}$ & Crudo 2 \\
\hline Limites: & & & & & & \\
\hline $\begin{array}{l}\text { Superior } \ldots \ldots \ldots \ldots \\
\text { Inferior } \ldots \ldots \ldots \ldots \ldots\end{array}$ & $\begin{array}{l}1,81 \\
1,72\end{array}$ & $\begin{array}{l}5,21 \\
5,09\end{array}$ & $\begin{array}{l}5,35 \\
5,17\end{array}$ & $\begin{array}{l}7,11 \\
6,89\end{array}$ & $\begin{array}{l}7,92 \\
7,76\end{array}$ & $\begin{array}{l}9,20 \\
0.02\end{array}$ \\
\hline$\varepsilon \%$ & 2,5 & 1,3 & $1, \bar{i}$ & 1,6 & 1,0 & 1,0 \\
\hline
\end{tabular}

Para los contenidos de sílice cuarzosa considerados, comprendidos entre 0 y $10 \%$, el error probable de la media aritmética oscila entre 1,0 y 2,5 , pudiendo aceptarse, en promedio, el valor de 1,5. La precisión o fidelidad del método, bien sea evaluada en función de estos errores, o en función de las desviaciones medias o errores típicos $\sigma_{m}$, es, pues, suficientemente aceptable en el caso estudiado. Lo mismo sucede con respecto a la precisión de cada uno de los resultados experimentales aislados.

\section{- Eonclusiones}

El método propuesto para la determinación de sílice libre en crudos y materias primas para la fabricación de cemento, puede adolecer, en ocasiones, de un error sistemático por defecto, de hasta un $0,18 \%$, para contenidos de cuarzo de hasta $10 \%$. Este error, debido a la diferente solubilidad de los distintos silicatos en ácido pirofosfórico, es insignificante, y ello indica que el método es suficientemente exacto.

Aparte de este error sistemático, el método, cuando se aplica en las condiciones que se especifican, puede estar afectado por otros errores accidentales, los cuales no dan lugar a resultados aberrantes. Los errores relativos por ciento de la media experimental respecto a la media verdadera, oscilan entre el 1 y el $2,5 \%$--en promedio $1,5 \%-$ y no son significativos, con lo cual la precisión del método puede considerarse aceptable.

\section{bibliografía}

1. Michaelis, W.: Memoria, marzo 1909.

2. Eilmann, T.: The influence of the fineness of cement raw mixes on their burnability, Proceedings of the third International Symposium on the Chemistry of Cement, London 1952, Paper 20.

3. Bogue, R. H.: La química del cemento Portland, pág. 169, Editorial Dossat, S. A., Madrid, 1952.

4. Dreyfus, J.: La Chimie des ciments, pág. 128, tomo I, Editions Eyrolles, París, 1950.

5. Hendrickx, J.: "IIe Point: Etat physico-chimique des melanges crues", Revue des Matériaux de Construction, julio 1947.

6. Sмidth, F. L. Co., A/S: Determination of content of sand in slurry, núm. 4611 B-3, 13-2-1952.

7. Ocella, E.: "La determinazione della silice liber cristalina", Il Cemento, 3, 1957.

8. Ocella, E.; Zurlo, N., y Frigerio G.: "Metodiche di analisi della silice libera cristalina", Il Cemento, 4, 1957.

9. Janssens, P. F.: "Etude microscopique, physique et chimique de la fraction sableuse des argiles. Dosage de la silice quartzeuse ou libre", Revue des Matériaux de Construction, núm. 436, enero 1952 .

10. Talvitie, N. A.: "Determination of quartz in presence of silicates using phosforic acid", Analytical Chemistry, vol. 23, nủm. 4, abril 1951.

11. Hess, R. L.: "Ensayo de arcillas", Cer. Age, 1 julio, 78 (7), 1962.

12. Fischer, M.: Les methodes estatistiques appliquées à la recherche scientifique, Presses Universitaires de France. París 1947. 